\title{
Study of Laser-Debonded GaN LEDs
}

\author{
Chung-Pui Chan, Jie Gao, Tai-Man Yue, Charles Surya, Member, IEEE, Alan Man-Ching Ng, \\ Aleksandra B. Djurišić, Peter Chow-Kee Liu, Senior Member, IEEE, and Ming Li
}

\begin{abstract}
Detailed investigations of laser-debonded GaN-based light-emitting diodes (LEDs) grown by metal-organic chemical vapor deposition (MOCVD) on sapphire substrates were reported. The debonded surface was roughened by photoelectrochemical (PEC) etching in a mixture of potassium hydroxide (KOH) and peroxydisulfate $\left(\mathrm{K}_{2} \mathrm{~S}_{2} \mathrm{O}_{8}\right)$ solution. The power for the laserassisted debonding process has been systematically optimized. The data show that as long as the laser power does not exceed the optimal value, there is no degradation in the current-voltage $(I-V)$ characteristics, the brightness, as well as the low-frequency noise properties of the devices. The roughness of the debonded surface is systematically varied using different etching times. Experimental results demonstrate strong dependencies of the luminous intensity of the device on the roughness of the debonded surface. A $60 \%$ improvement in the luminous intensity of the debonded and roughened LED compared to the original on-sapphire device was observed. This increase in the extraction efficiency is attributed to the reduction in the total internal reflection at the roughened GaN/air interface.
\end{abstract}

Index Terms-Gallium nitride, hexagonal pyramid, laser debonding, light emitting diodes, low-frequency noise, roughening.

\section{INTRODUCTION}

$\mathbf{T}$ HE III-nitrides are materials of choice for the development of many important optoelectronic and electronic devices [1]-[4]. Their large direct band gaps have been utilized for the development of light-emitting diodes (LEDs) for applications in full-color displays, traffic signals, and automotive lighting. Recent development of GaN LEDs demonstrates an efficacy of $74 \mathrm{~lm} / \mathrm{W}$ for packaged white-light LEDs [5]. It has been pointed out that further enhancements in the efficacy will have to be achieved for the GaN LEDs to be widely used in general lighting. Hence, it is crucial that the overall efficiency of the device be improved.

Sapphire is the most commonly used substrate for the growth of GaN-based LEDs due to its relative low price and its stability at high temperatures. A number of issues arise due to the use of sapphire substrates, including thermal dissipation, current

Manuscript received February 9, 2006; revised May 15, 2006. This work was supported in part by the Research Grants Council of Hong Kong under Projects PolyU 5130/01E, PolyU 5226/03E, and PolyU 5236/03E, and in part by the Hong Kong Polytechnic University under a University Research Grant. The review of this paper was arranged by Editor L. Lunardi.

C. P. Chan, J. Gao, and C. Surya are with the Department of Electronic and Information Engineering and Photonics Research Centre, Hong Kong Polytechnic University, Hong Kong (e-mail: ensurya@polyu.edu.hk).

T. M. Yue is with the Department of Industrial and Systems Engineering and Advanced Manufacturing Technology Research Center, Hong Kong Polytechnic University, Hong Kong.

A. M. C. $\mathrm{Ng}$ and A. B. Djurišić are with the Department of Physics, University of Hong Kong, Hong Kong.

P. C.-K. Liu and M. Li are with ASM Pacific Technology Ltd., Hong Kong. Digital Object Identifier 10.1109/TED.2006.881008 crowding effects, and low extraction efficiency $\eta_{\mathrm{ex}}$ [6]. Much effort had been devoted for the improvement of $\eta_{\mathrm{ex}}$ in the past decade. It is known that the geometric shape of the device surface plays a critical role in determining the efficiency in the escape of light from the device. Die shaping in the form of an inverted truncated cone has shown to significantly improve the extraction efficiency of LEDs [7]-[9]. Unfortunately, this is not possible for GaN-based LEDs grown on sapphire substrates due to the hardness of sapphire.

Fujii et al. demonstrated that conelike structures may be formed on $\mathrm{n}-\mathrm{GaN}$ surface by photoenhanced chemical wet etching with a potassium hydroxide $(\mathrm{KOH})$ solution and the light extraction efficiency of the roughened LEDs is significantly enhanced by 2-3 times [10]. However, this is not achieved without penalty. The removal of the undoped GaN using the inductively coupled plasma (ICP) etching technique may induce radiation damage on the LED, which may lead to device reliability problems. Other techniques such as the use of transparent conductive layers for current spreading in the p-contact [11], [12] and the deposition of reflective metallic layers on the GaN surface as light reflectors in flip-chip bonded devices [13] have also proved to be successful in increasing the light extraction efficiency to a certain extent. Due to the large difference in the refractive indexes between $\mathrm{GaN}$ and the dielectric encapsulation, which is typically used in LED packaging, the extraction efficiency of the conventional topemitted LEDs remains to be quite poor [14]. It has been shown that while the use of the flip-chip bonding technique may significantly improve the current spreading and the efficiency of thermal dissipation for GaN LEDs on sapphire, the improvement in the extraction efficiency of the devices remains to be quite limited. Using the reported values of the refractive indexes for $\mathrm{GaN}\left(n_{\mathrm{GaN}}=2.4\right)$ and the sapphire substrate $\left(n_{\mathrm{Sa}}=1.8\right)$, a critical angle of $48.5^{\circ}$ and a transmission efficiency of $13.8 \%$ across the GaN/sapphire interface can be obtained. The majority of the emitted photons are internally reflected and eventually lost through various reabsorption processes.

In this paper, we investigate the improvement in $\eta_{\mathrm{ex}}$ by laser-assisted debonding of the sapphire substrate, followed by photoelectrochemical (PEC) etching, to give high surface roughness at the debonded GaN surface. Studies have shown that a roughened surface has the potential of substantially reducing the total internal reflection. The optical properties of the devices are characterized by detailed examination of the electroluminescence (EL) spectra before and after the laser debonding process for different etching times. The results are correlated to the surface morphology of the etched $\mathrm{GaN}$ surface. 


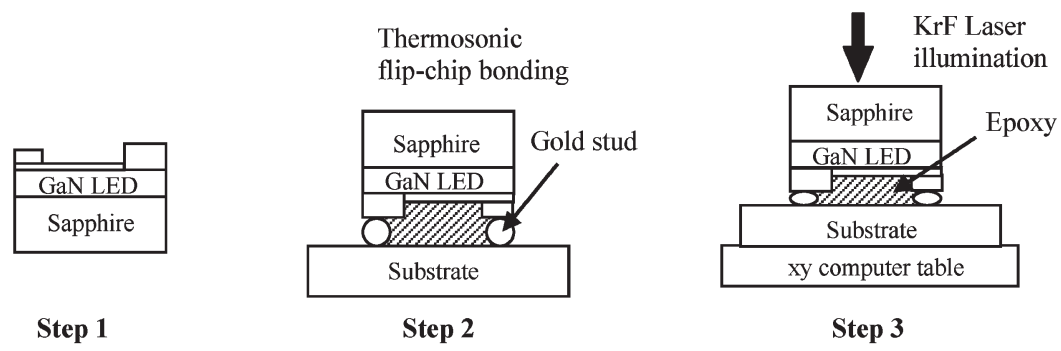

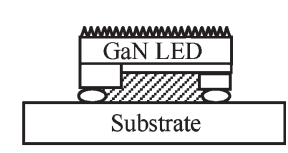

Step 5

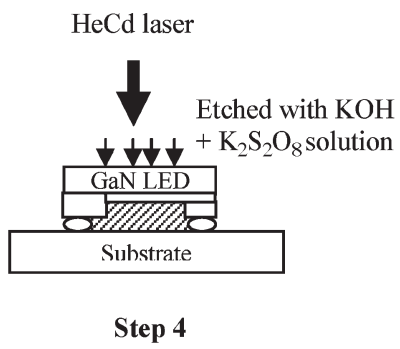

Fig. 1. Laser debonding and surface roughening processes.

\section{EXPERIMENT}

Standard multiple quantum well (MQW) LED structures were grown by metal-organic chemical vapor deposition (MOCVD), and the structure consists of a $1-\mu \mathrm{m}$-thick undoped GaN layer, followed by a $1-\mu \mathrm{m}$-thick n-type layer, a GaN/InGaN MQW, and a 1- $\mu$ m-thick p-type layer. Ohmic contacts to the $n$ - and p-layers are fabricated on the same side of the devices. The LED structures were then flip-chip bonded to a silicon substrate using an AD819-11TS thermosonic flip-chip bonder. Laser-assisted debonding of the sapphire substrates was accomplished using a Lambda Physik excimer laser operating at a wavelength of $\lambda=248 \mathrm{~nm}$. At such wavelength, the sapphire substrate is transparent, and the radiation is absorbed by a thin GaN layer with a thickness of about $200 \mathrm{~nm}$ at the GaN/sapphire interface. Wong et al. [15] showed that when $\mathrm{GaN}$ is irradiated with a $\mathrm{KrF}$ excimer laser at a fluence of $400 \mathrm{~mJ} \cdot \mathrm{cm}^{-2}$ or above, local heating of a thin layer of $\mathrm{GaN}$ at the sapphire/GaN interface results in the decomposition of the material into metallic $\mathrm{Ga}$ and $\mathrm{N}$ gas. This will serve as a sacrificial layer for the debonding process, and the exact thickness of this layer is found to depend on the actual laser power used. Since the melting point of metallic $\mathrm{Ga}$ is about $30{ }^{\circ} \mathrm{C}$, it is found that when the material is warmed to about $40{ }^{\circ} \mathrm{C}-50{ }^{\circ} \mathrm{C}$, the $\mathrm{GaN}$ film can be easily separated from the sapphire substrate. The debonding/PEC wet-etching process is illustrated in Fig. 1.

We investigated the roughening of the debonded surface of a device by PEC etching using a $2 \mathrm{M} \mathrm{KOH}$ and $0.05 \mathrm{M}$ peroxydisulfate $\left(\mathrm{K}_{2} \mathrm{~S}_{2} \mathrm{O}_{8}\right)$ solution. A 16-mW HeCd laser emitting a coherent light at $325 \mathrm{~nm}$ was used as the light source. The surface morphology is monitored with a scanning electron microscope (SEM) and a Veeco NT 8000 optical profiler over a large area. The luminance/current-voltage $(I-V)$ characteristics were measured using a Keithley 2400 sourcemeter and an Oriel MS 260I monochromator with an integrating sphere and charge-coupled device (CCD) detector.

\section{RESULTS AND DISCUSSIONS}

To optimize the laser power, we investigated the amount of $\mathrm{GaN}$ decomposed due to a single laser pulse, using a $\mathrm{KrF}$ excimer laser, as a function of laser energy density, which varies from $100 \mathrm{~mJ} \cdot \mathrm{cm}^{-2}$ to $1 \mathrm{~J} \cdot \mathrm{cm}^{-2}$. The decomposition of the GaN material is attributed to the absorption of photon energy, which produces a local heating at the GaN/sapphire interface. Chu et al. [16] showed that GaN breaks down into metallic $\mathrm{Ga}$ and $\mathrm{N}$ gas when the local temperature exceeds $1000{ }^{\circ} \mathrm{C}$ during the laser illumination process. The amount of $\mathrm{GaN}$ decomposed in a single laser pulse is directly proportional to the thickness of the sacrificial layer, which is measured using a profilometer. Our study shows that no detectable material removal is obtained when the laser energy density falls below $300 \mathrm{~mJ} / \mathrm{cm}^{2}$. For higher energy densities, some metallic $\mathrm{Ga}$ droplets are observed at the interfacial region, and a fluence of $400 \mathrm{~mJ} \cdot \mathrm{cm}^{-2}$ corresponds to the minimum laser power that can achieve a clean separation of the sapphire substrate from the GaN layer. The corresponding thickness of the sacrificial layer is found to be $180 \mathrm{~nm}$.

Theoretical analysis of the temperature distribution within the GaN LED during the laser illumination process can be performed by solving the following differential equation using the finite-element technique:

$$
\frac{\partial T}{\partial t}=\frac{\alpha}{\rho C_{P}} I(z, t)+\frac{1}{\rho C_{P}} \frac{\partial}{\partial z}\left(\kappa \frac{\partial T}{\partial t}\right)
$$

Here, $\rho$ is the density, $C_{P}$ is the specific heat, $\kappa$ is the thermal conductivity, $\alpha$ is the optical absorption, and $z$ is denoted as the distance from the GaN/sapphire interface. The incident power density is given by $I(z, t)=I_{0}(t)(1-R) e^{-\alpha z}$, where $I_{0}(t)$ is the output laser power density and $R$ is the GaN reflectivity. For an energy density of $400 \mathrm{~mJ} \cdot \mathrm{cm}^{-2}$ and a pulse duration of $25 \mathrm{~ns}$, the solution for $T$ is shown in Fig. 2. The results indicate that the maximum temperature drops rapidly from 1340 to 


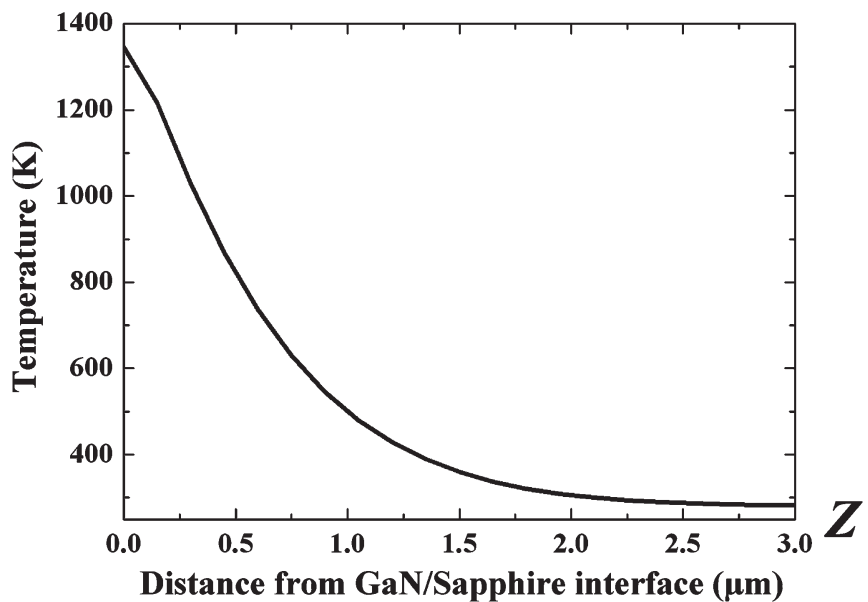

Fig. 2. Temperature variation of the irradiated GaN film using a laser energy density of $400 \mathrm{~mJ} \cdot \mathrm{cm}^{-2}$ at a time of $25 \mathrm{~ns}$ from the instance of illumination, as a function of the distance from the GaN/sapphire interface.

below $1273 \mathrm{~K}$ within $200 \mathrm{~nm}$ from the interface, which is in good agreement with our experimental measurement of the thickness of the sacrificial layer.

To investigate the degradation of the device properties due to the laser illumination process, we have studied the $I-V$ and low-frequency noise properties of two devices being debonded using two different energy densities of 400 (device I) and $500 \mathrm{~mJ} \cdot \mathrm{cm}^{-2}$ (device II). In Fig. 3, we present the $I-V$ characteristics before and after laser debonding of the devices. Experimental data show that there is no degradation in the $I-V$ characteristics for device I before and after laser-assisted debonding. However, when a fluence of $500 \mathrm{~mJ} \cdot \mathrm{cm}^{-2}$ is used, substantial degradation in the $I-V$ properties for device II is observed. Tavernier and Clarke [17] pointed out that damagefree GaN films can be obtained when the laser energy density is just sufficient to decompose the $\mathrm{GaN}$ material at the interface. This facilitates debonding without generating any appreciable vapor pressure. It is, therefore, believed that when excessive laser power is used, the material degradation arises from the large amount of nitrogen gas emitted from the decomposition of the excessive amount of $\mathrm{GaN}$, leading to the mechanical fracture of the GaN film [18].

The material quality of the device is also investigated by the measurement of low-frequency excess noise. This is because the current noise power spectral density $S_{I}(f)$ of the device in low-frequency regime is found to be directly proportional to the trap density $N_{T}$ in the active region. Based on the thermal activation model, flicker noise arises from the thermally activated trapping and detrapping of carriers by localized states with activation energy $E$. The current noise power spectral density is given by

$$
\begin{aligned}
S_{I}(f)=4 \frac{V^{2}}{R_{d}^{2} N^{2}} \int_{x} \int_{y} \int_{z} \int_{E} N_{T}(x, y, z, E) \\
\times \frac{\tau}{1+\omega^{2} \tau^{2}} d x d y d z d E
\end{aligned}
$$

in which $N_{T}(E)$ is the trap density per unit energy, $V$ is the dc voltage applied across the device, $R_{d}$ is the device resistance,
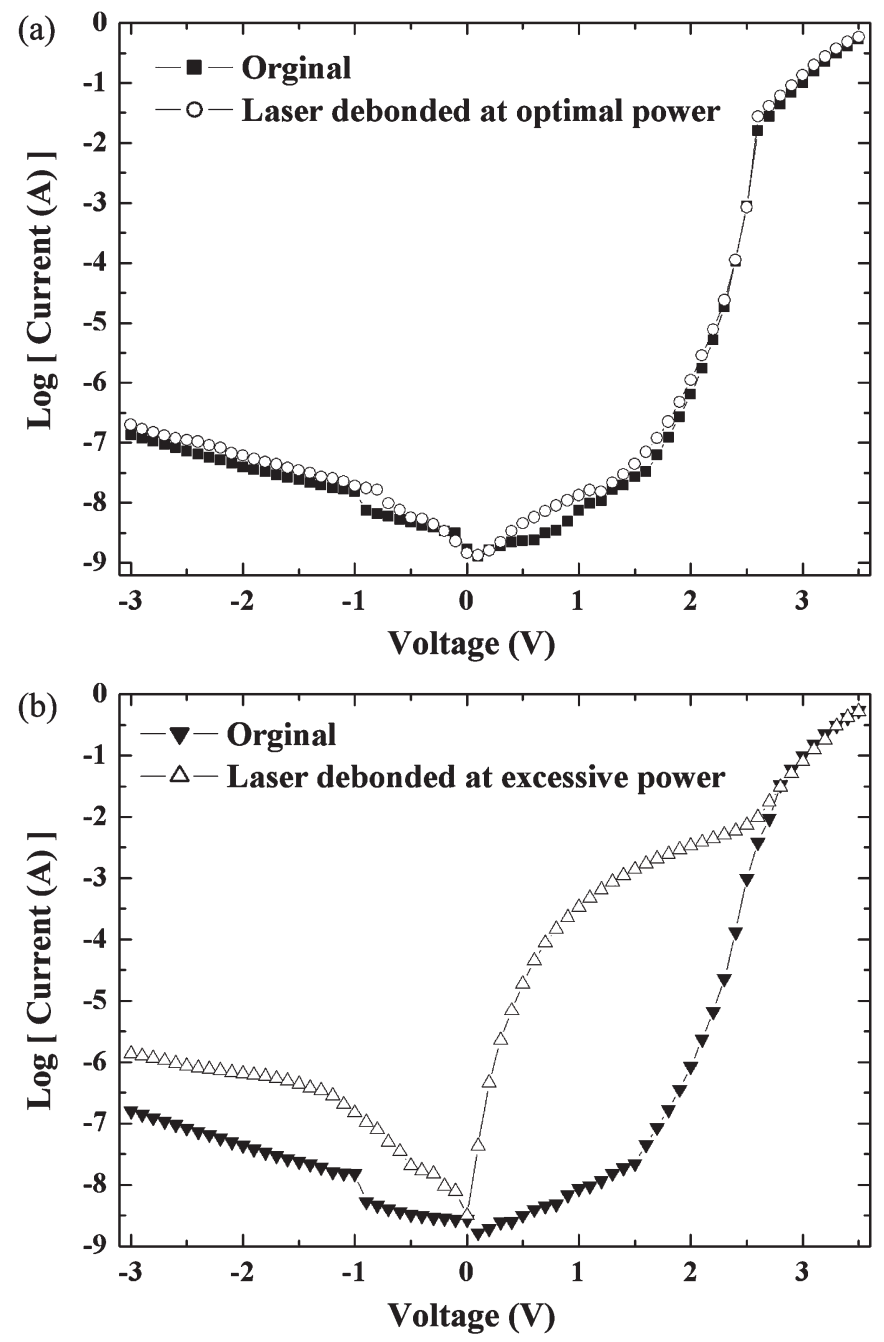

Fig. 3. $I-V$ characteristics of the LED before and after laser debonding at (a) a optimal laser energy density of $400 \mathrm{~mJ} \cdot \mathrm{cm}^{-2}$ and (b) a laser energy density of $500 \mathrm{~mJ} \cdot \mathrm{cm}^{-2}$.

$N$ is the total number of carriers in the active region, and $\tau$ is the thermally activated fluctuation time constant given by

$$
\tau=\tau_{0} \exp \left(\frac{E}{k_{B} T}\right)
$$

where $\tau_{0}$ is the inverse attempt rate and $k_{B}$ is Boltzmann's constant. The Lorentzian in (2) peaks sharply at $E_{p}=$ $-k_{B} T \ln \left(\tau_{0} \omega\right)$, indicating that the $S_{I}(f)$ observed at a particular frequency $f$ and temperature $T$ is caused by the capture and emission of carriers by localized states with activation energy $E_{p}$. If $N_{T}(E)$ remains constant at around $E_{p}$, then $S_{I}(f)$ is shown to be proportional to $1 / f^{\gamma}$, where $\gamma=1$. On the other hand, it has been shown that if $N_{T}(E)$ increases in $E$, then $\gamma>$ 1 and if $N_{T}(E)$ decreases in $E$, then $\gamma<1$. The experimental values of $S_{I}(f)$ measured from devices I and II before and after laser debonding are shown in Fig. 4. The data indicate that room-temperature low-frequency fluctuations are dominated by flicker noise over the frequency range of our measurements. No g-r bumps are observed in our data. Curves A1 and A2, which are measured from device I, clearly show that there is basically no increase in $S_{I}(f)$ after laser debonding. However, 


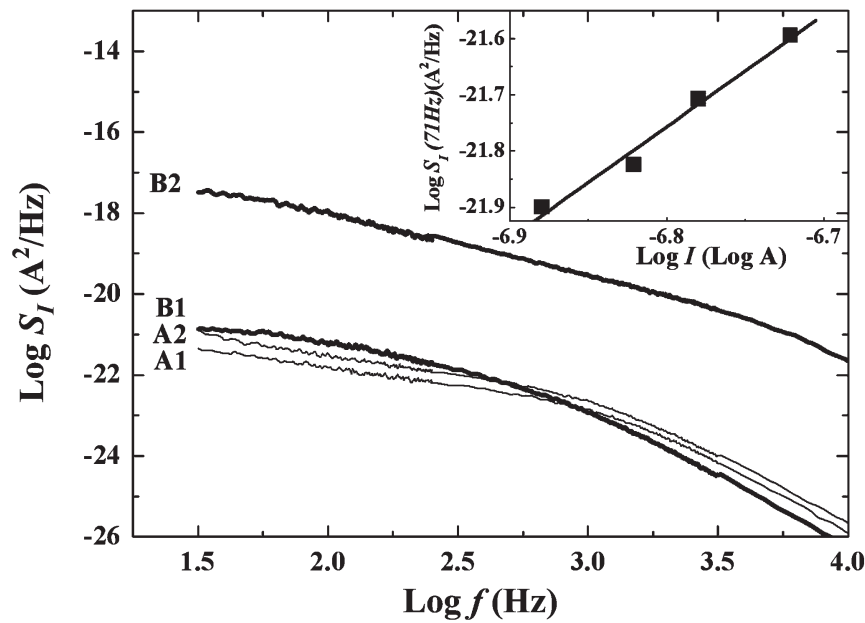

Fig. 4. Room-temperature $S_{I}(f)$ measured from devices I (curves A1 and A2) and II (curves B1 and B2). Curves A1 and B1 represent the data taken before laser debonding, and curves $\mathrm{A} 2$ and $\mathrm{B} 2$ represent the data taken after laser debonding.

device II indicates substantial increase in room-temperature $S_{I}(f)$ before and after laser debonding as shown in curves B1 and B2, respectively. The result stipulates substantial increase in the defect density for device II.

We performed electrodeless PEC etching of the debonded surface of the GaN LEDs using a mixture of $2 \mathrm{M} \mathrm{KOH} /$ $0.05 \mathrm{M} \mathrm{K}_{2} \mathrm{~S}_{2} \mathrm{O}_{8}$ solution. Undoped $\mathrm{GaN}$ is chemically inert, and to the best of our knowledge, there is, so far, little report on the wet etching of undoped GaN. In the previous investigation, ICP etching was employed to remove the undoped layer so as to expose the underlying $\mathrm{n}-\mathrm{GaN}$, and the $\mathrm{KOH}$ solution was then used to roughen the $\mathrm{n}-\mathrm{GaN}$ surface. However, the damages induced by the ICP etching technique on the GaN material and their devices have aroused much concern [19]-[21]. In our studies, we demonstrate that the PEC etching technique, at the mentioned conditions, can be utilized to roughen an undoped GaN layer to form hexagonal pyramid structures. This leads to an increase in the light extraction efficiency of the LED. The success of this PEC technique would not only open up another alternative for $\mathrm{GaN}$ etching but also eradicate any risks of material damages associated with ICP etching.

The debonded surface consists of unintentionally doped GaN material. The etching of GaN is a two-step process: In the first step, $\mathrm{GaN}$ is oxidized following

$$
2 \mathrm{GaN}+6 \mathrm{OH}^{-}+6 \mathrm{~h}^{+} \longrightarrow \mathrm{Ga}_{2} \mathrm{O}_{3}+3 \mathrm{H}_{2} \mathrm{O}+\mathrm{N}_{2}
$$

which indicates that the presence of holes is crucial for the reaction. The holes are typically supplied by a cathode in a conventional electrochemical cell. This is followed by the dissolution of the $\mathrm{Ga}_{2} \mathrm{O}_{3}$ layer. For electrodeless PEC etching, the use of a mixture of $\mathrm{KOH}$ and $\mathrm{K}_{2} \mathrm{~S}_{2} \mathrm{O}_{8}$ eliminates the need for a physical connection between the sample and the cathode, as $\mathrm{K}_{2} \mathrm{~S}_{2} \mathrm{O}_{8}$ is an oxidizing agent that facilitates the generation of a hole in the valence band. The illumination of the etched surface by the $\mathrm{HeCd}$ laser greatly enhances the etch rate.

In Fig. 5, we present some SEM pictures of the debonded $\mathrm{GaN}$ surface after being etched in the $2 \mathrm{M} \mathrm{KOH} / 0.05 \mathrm{M}$ (a)

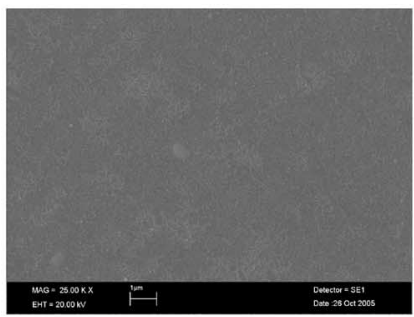

(c)

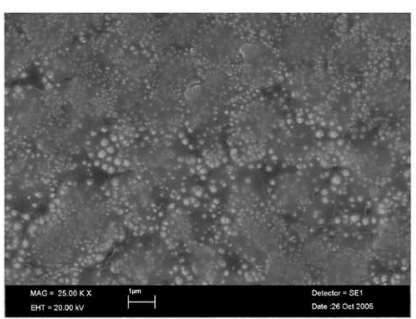

(e)

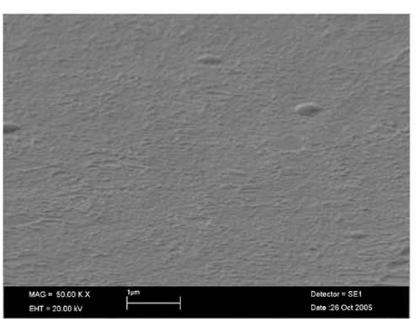

(g)

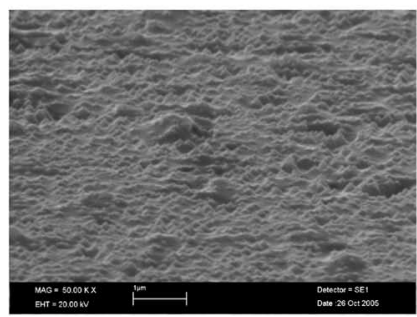

(b)

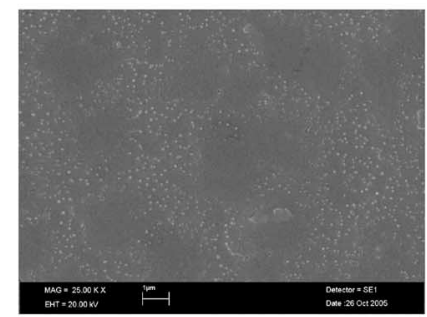

(d)

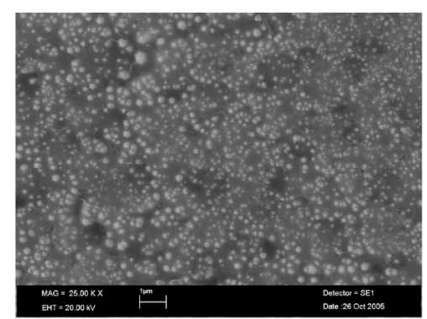

(f)

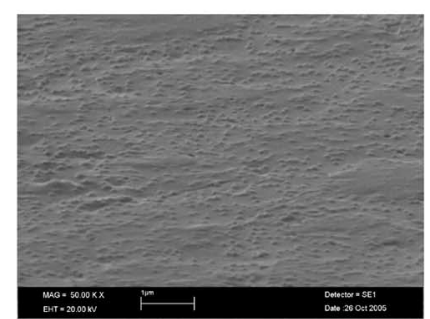

(h)

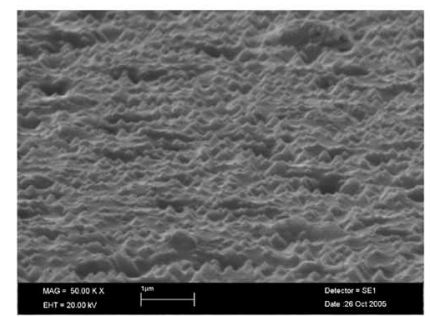

Fig. 5. SEM pictures showing the etched surface morphologies with different etching times. (a), (b), (c), and (d) are the top view and (e), (f), (g) and (h) are taken at an inclined angle of 30 degrees from the parallel direction of the roughened surface.

$\mathrm{K}_{2} \mathrm{~S}_{2} \mathrm{O}_{8}$ mixture for different durations, which clearly indicate increasing surface roughness with etching time. In Table I, we indicate the root-mean-square (rms) roughness of the etched $\mathrm{GaN}$ surface, as measured by the optical profiler. It is clear from Figs. 5(a) and 5(e) that the as-debonded surface exhibits relatively smooth morphology and is free from any surface cracking. The 15-min etched sample [Fig. 5(b) and 5(f)] shows some hillock-like structures on the etched surface, and each of them has been correlated to be a threading dislocation center [22], [23]. Hexagonal pyramid structures are formed for etch times that are greater than 30 min [Figs. 5(c) and 5(g)]. Significant roughening of the $\mathrm{GaN}$ surface is seen with an etch time of 40 min [Figs. 5(d) and 5(h)], which reveals larger pyramidal structures with a size of $100-400 \mathrm{~nm}$. This may potentially lead to significant improvement in the light extraction efficiency of the LEDs.

The emission spectra for devices with different etch times, in which curves $\mathrm{A}, \mathrm{C}$, and D correspond to etch times of 15 , 
TABLE I

RMS ROUGHNESS OF THE ETCHED GaN SURFACE

\begin{tabular}{|c|c|}
\hline Etching time (mins) & RMS roughness (nm) \\
\hline 0 (As debonded) & 8.61 \\
\hline 15 & 43.54 \\
\hline 30 & 165.41 \\
\hline 40 & 238.10 \\
\hline
\end{tabular}

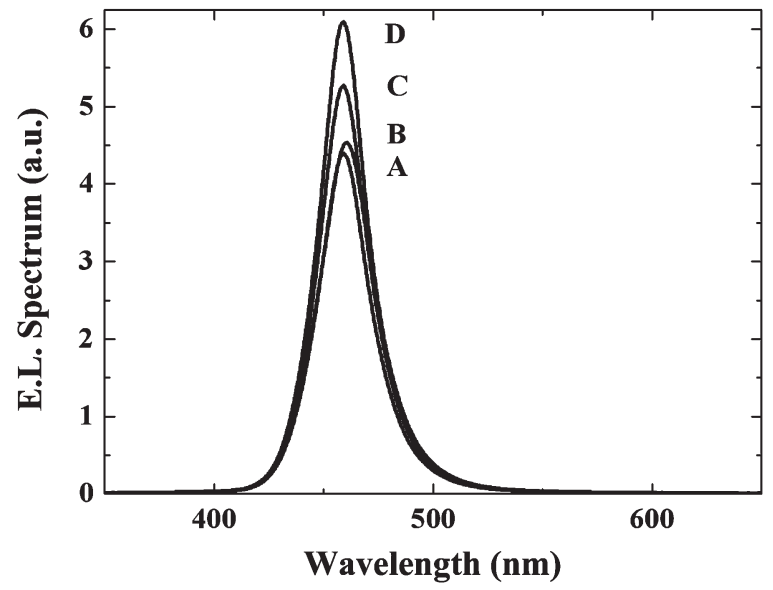

Fig. 6. EL spectra of the GaN LEDs with different etching times.

30, and 40 min, respectively, are shown in Fig. 6. Curve B is the control, corresponding to the emission spectrum of the device before laser debonding. The experimental data indicate substantial enhancements in the EL spectra for laser-debonded devices with etching times greater than $30 \mathrm{~min}$. Using an integrating sphere, we obtain the luminous intensity of the devices, and the results are shown in Fig. 7 as a function of the rms roughness.

The separation of the GaN films from the sapphire substrates eliminates any total internal reflection that might have taken place at the GaN/sapphire interface. However, the data show that there is no improvement in the overall extraction efficiency unless the debonded surface is roughened by PEC etching. This is because of the large difference in the refractive index between the $\mathrm{GaN}$ and air, which is even greater than that between the $\mathrm{GaN}$ and the sapphire substrate. This explains the initial drop in the light output for device A, as shown in Figs. 6 and 7. The improvements in light extraction efficiency in the roughened devices are due to the fact that when there is an incident light ray on the pyramid/air interface, it may either transmit across the interface or be totally internally reflected within the pyramid structure. In this case, the reflected ray undergoes multiple reflections within the pyramid with an increasing angle of incidence with each reflection; eventually, the light ray will exit the pyramid.

The experimental results in Fig. 7 demonstrate an improvement of over $60 \%$ in the luminous intensity after $40 \mathrm{~min}$ of PEC etching. Fig. 8 indicates that the increase in light extraction due to surface roughening can be observed at different current levels, and it is interesting to note that the data in Fig. 8 do not show any trend in saturation. This indicates the possibility of further improvements in the luminous intensity. To achieve this, the design of the LED structure may have to be modified

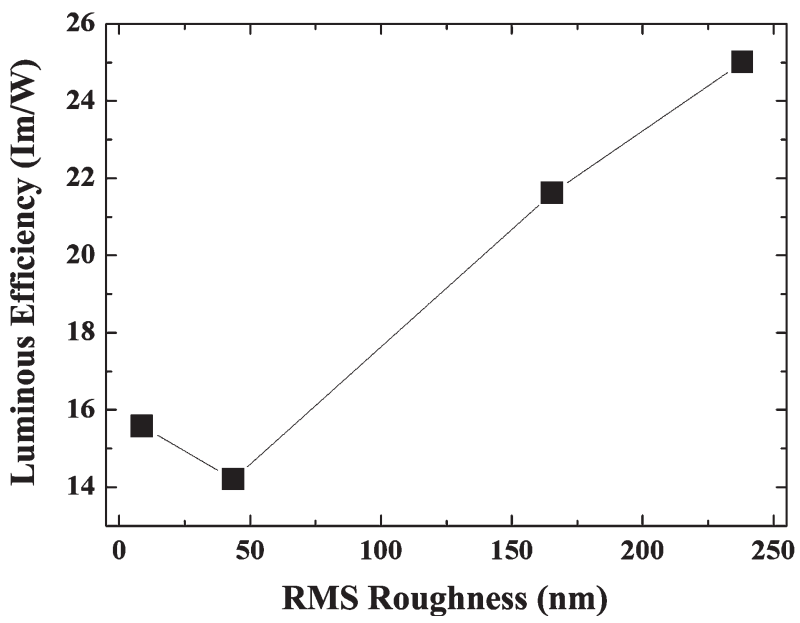

Fig. 7. Luminous intensity as a function of the rms roughness of the debonded GaN surface.

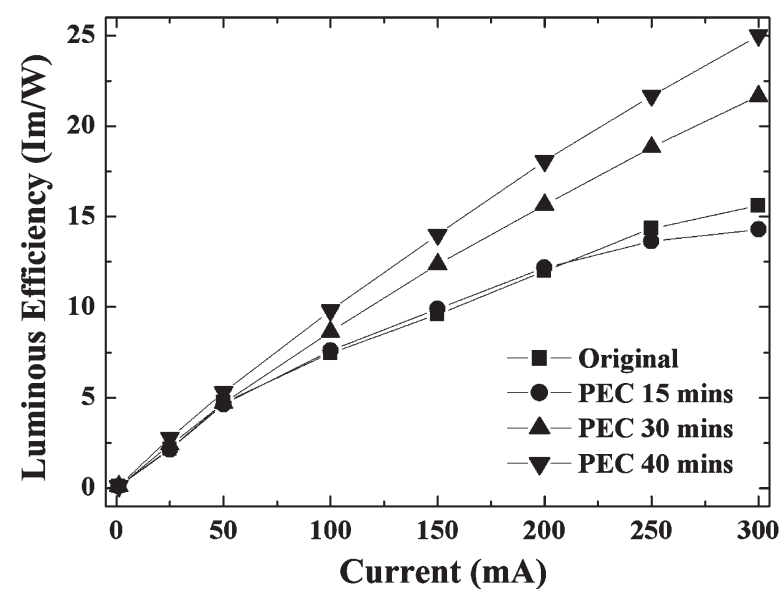

Fig. 8. Light output intensity as a function of injection current at different etching times.

such that an n-doped GaN layer is used in place of the undoped GaN layer in the LED structure. This will greatly enhance the etching rate and thereby substantially reduce the etching time needed for the formation of the hexagonal pyramids.

In addition, a slight peak shift from 460.6 (device B) to $459.2 \mathrm{~nm}$ (devices A, C, and D) in the EL spectra is observed for such devices after laser debonding. Two possible mechanisms may give rise to such a shift, namely 1) MQW structures intermixing due to thermal annealing as well as strain relaxation after laser debonding, and 2) strain relaxation of the GaN film due to the separation from the sapphire substrate. Based on the experimental results, strain relaxation would be more reasonable in this case since the results on thermal analysis in Fig. 2 show that the MQW structure, which is roughly $2.5 \mu \mathrm{m}$ away from the sapphire/ $\mathrm{GaN}$ interface, remained basically at room temperature during the debonding process. In contrast, the high-resolution X-ray diffraction (XRD) measurements in our recent studies show that both in-plane and out-plane strains are released after the GaN LED is separated from its sapphire substrate. This result corroborates with the study by Lee $e t$ al. on the strain properties in $\mathrm{GaN}$ films, which have indicated that 
the strain relaxation of $\mathrm{GaN}$ film would lead to a decrease in piezoelectric field, thus resulting in a blue shift in the emission spectra [24].

\section{CONCLUSION}

We have studied the optoelectronic properties of laserdebonded GaN-based LEDs grown on sapphire substrates. We demonstrated that there is no degradation in the device properties, as long as the optimal energy density for the laser pulse is used. We have also demonstrated significant improvements in the extraction efficiency for the debonded devices when PEC etching is applied to the debonded $\mathrm{GaN}$ surface. The etching of the $\mathrm{GaN}$ results in the formation of hexagonal pyramidal structures on the GaN surface, resulting in substantial roughening of the GaN surface and, hence, reduction in the total internal reflection at the GaN/air interfaces. An improvement close to $60 \%$ in the luminous intensity of the device is observed.

\section{REFERENCES}

[1] A. Krost and A. Dadgar, "GaN-based optoelectronics on silicon substrates," Mater. Sci. Eng. B, vol. 93, no. 1/3, pp. 77-84, May 2002.

[2] M. Umeno, T. Egawa, and H. Ishikawa, "GaN-based optoelectronic device on sapphire and Si substrates," Mater. Sci. Semicond. Process., vol. 4, no. 6, pp. 459-466, Dec. 2001.

[3] J. C. Carrano, T. Li, P. A. Grudowski, C. J. Eiting, R. D. Dupuis, and J. C. Campbell, "Comprehensive characterization of metal-semiconductor-metal ultraviolet photodetectors fabricated on single-crystal GaN," J. Appl. Phys., vol. 83, no. 11, pp. 6148-6160, Jun. 2000.

[4] T. D. Moustakas, E. Iliopoulos, A. V. Sampath, H. M. Ng, D. Doppalapudi, M. Misra, D. Korakakis, and R. Singh, "Growth and device applications of III-nitrides by MBE,” J. Cryst. Growth, vol. 227, pp. 13-20, Jul. 2001.

[5] T. Fujii, Y. Gao, R. Sharma, E. L. Hu, S. P. DenBaars, and S. Nakamura, "Increase in the extraction efficiency of GaN-based light-emitting diodes via surface roughening," Appl. Phys. Lett., vol. 84, no. 6, pp. 855-857, Feb. 2004.

[6] C. R. Miskys, M. K. Kelly, O. Ambacher, and M. Stutzmann, "Freestanding GaN-substrates and devices," Phys. Stat. Sol. C, vol. 0, no. 6, pp. 1627-1650, 2003.

[7] E. F. Schubert, Light-Emitting Diodes. Cambridge, U.K.: Cambridge Univ. Press, 2003.

[8] E. H. Park, J. H. Kim, T. K. Yoo, and Y. S. Kwon, "Bell-shaped light emitting diodes (BS-LED) with a 45 degree corner reflector, deep sidewall, and microlens," in Proc. SPIE-The International Society for Optical Engineering, 2002, vol. 4641, pp. 19-30.

[9] S. S. Schad, M. Scherer, M. Seyboth, and V. Schwegler, "Extraction efficiency of GaN-based LEDs," Phys. Status Solidi A, vol. 188, no. 1, pp. 127-130, Nov. 2001.

[10] T. Fujii, A. David, Y. Gao, M. Iza, S. P. DenBaars, E. L. Hu, C. Weisbuch, and S. Nakamura, "Cone-shaped surface GaN-based lightemitting diodes," Phys. Stat. Sol. C, vol. 2, no. 7, pp. 2836-2840, 2005.

[11] D. W. Kim, Y. J. Sung, J. W. Park, and G. Y. Yeom, "A study of transparent indium tin oxide (ITO) contact to p-GaN," Thin Solid Films, vol. 398/399, pp. 87-92, Nov. 2001

[12] C. Huh, H. S. Kim, S. W. Kim, J. M. Lee, D. J. Kim, I. H. Lee, and S. J. Park, "InGaN/GaN multiple quantum well light-emitting diodes with highly transparent Pt thin film contact on p-GaN," J. Appl. Phys., vol. 87, no. 9, pp. 4464-4466, May 2000.

[13] R. Zheng and T. Taguchi, "Optical design of large-area GaN-based LEDs," in Proc. SPIE-The International Society for Optical Engineering, 2003, vol. 4996, pp. 105-112.

[14] T. Baba, K. Inoshita, H. Tanaka, J. Yonekura, M. Ariga, A. Matsutani, T. Miyamoto, F. Koyama, and K. Iga, "Strong enhancement of light extraction efficiency in GaInAsP 2-D-arranged microcolumns," J. Lightw. Technol., vol. 17, no. 11, pp. 2113-2120, Nov. 1999.

[15] W. S. Wong, Y. Cho, E. R. Weber, T. Sands, K. M. Yu, J. Kruger, A. B. Wengrow, and N. W. Cheung, "Structural and optical quality of GaN/metal/Si heterostructures fabricated by excimer laser lift-off," Appl. Phys. Lett., vol. 75, no. 13, pp. 1887-1889, Sep. 1999.
[16] C. F. Chu, F. Lai, J. T. Chu, C. C. Yu, C. F. Lin, H. C. Kuo, and S. C. Wang, "Study of GaN light-emitting diodes fabricated by laser liftoff technique," J. Appl. Phys., vol. 95, no. 8, pp. 3916-3922, Apr. 2004.

[17] P. R. Tavernier and D. R. Clarke, "Mechanics of laser-assisted debonding of films," J. Appl. Phys., vol. 89, no. 3, pp. 1527-1536, Feb. 2001.

[18] J. Xu, R. Zhang, Y. P. Wang, X. Q. Xiu, B. Shen, S. L. Gu, Y. Shi, Z. G. Liu, and Y. D. Zheng, "Preparation of large area freestanding GaN by laser lift-off technology," Mater. Lett., vol. 56, no. 1, pp. 43-46, Sep. 2002.

[19] S. Y. Han, H. S. Yang, K. H. Baik, S. J. Pearton, and F. Ren, "Role of ion energy and flux on inductively coupled plasma etch damage in InGaN/GaN multi quantum well light emitting diodes," Jpn. J. Appl. Phys., vol. 44, no. 10, pp. 7234-7237, 2005.

[20] R. J. Shul, L. Zhang, A. G. Baca, C. G. Willison, J. Han, S. J. Pearton, and F. Ren, "Inductively coupled plasma-induced etch damage of GaN p-n junctions," J. Vac. Sci. Technol. A, Vac. Surf. Films, vol. 18, no. 4, pp. 1139-1143, 2000.

[21] K. J. Choi, H. W. Jang, and J. L. Lee, "Observation of inductively coupledplasma-induced damage on n-type GaN using deep-level transient spectroscopy," Appl. Phys. Lett., vol. 82, no. 8, pp. 1233-1235, Feb. 2003.

[22] C. Youtsey, L. T. Romano, R. J. Molnar, and I. Adesida, "Rapid evaluation of dislocation densities in n-type GaN films using photoenhanced wet etching," Appl. Phys. Lett., vol. 74, no. 23, pp. 3537-3539, Jun. 1999.

[23] N. G. Weimann, L. F. Eastman, D. Doppalapudi, H. M. Ng, and T. D. Moustakas, "Scattering of electrons at threading dislocations in GaN," J. Appl. Phys., vol. 83, no. 7, pp. 3656-3659, Apr. 1998.

[24] K. S. Lee, C. Huh, J. M. Lee, E. J. Kang, and S. J. Park, "Electrical and optical characteristics of InGaNÕGaN microdisk LEDs," Electrochem. Solid-State Lett., vol. 8, no. 3, pp. G68-G70, 2005.

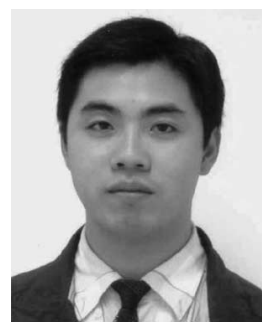

Chung-Pui Chan received the B.Eng. and M.Phil. degrees from the Department of Industrial and Systems Engineering, Hong Kong Polytechnic University, Hong Kong, in 1999 and 2002, respectively. He is currently working toward the Ph.D. degree at Hong Kong Polytechnic University, under Prof. C. Surya and Prof. T. M. Yue.

His research interests include laser material processing, and fabrication and characterization of III-nitride thin films.

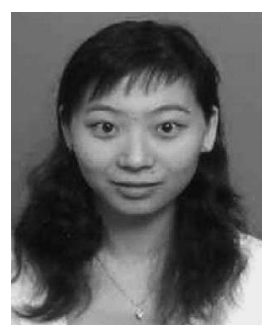

Jie Gao received the B.Eng. degree in 2003 from the Department of Electronic Engineering, Peking University, Beijing, in 2003 and the M.Phil. degree from Hong Kong Polytechnic University, Hong Kong, under Prof. C. Surya.

Her research interests include etching techniques for the fabrication process and characterization of III-V nitride thin films.

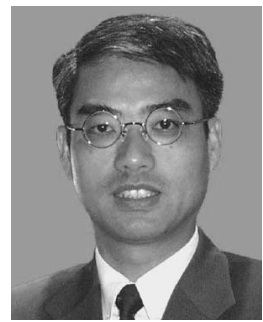

Tai-Man Yue received the B.Sc. and Ph.D. degrees from Southampton University, Southampton, U.K.

$\mathrm{He}$ was a Senior Materials Scientist with $\mathrm{Hi}-\mathrm{Tec}$ Metals R\&D Ltd., U.K. In 1991, he joined Hong Kong Polytechnic University, Hong Kong, where he is currently a Professor in the Department of Industrial and Systems Engineering. His research interests include laser material processing, processing of composite materials, and pulse electroforming.

Dr. Yue is a Chartered Engineer. 


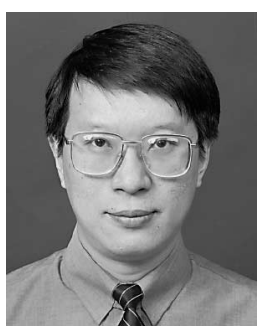

Charles Surya (S'86-M'87) received the Ph.D. degree in electrical engineering from the University of Rochester, Rochester, NY, in 1987.

From 1987 to 1994 , he was a Faculty Member with Northeastern University, Boston, MA. In 1994, he joined Hong Kong Polytechnic University, Hong Kong. His research interests include III-nitride-based devices and the study of fluctuation phenomena in semiconductor materials and devices.

Dr. Surya served as the Chairman of the IEEE Electron Device Society Hong Kong Chapter during 1996-1997.

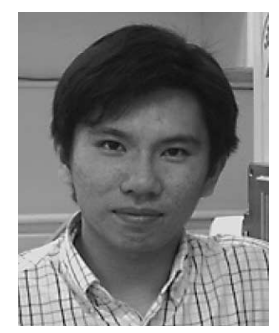

Alan Man-Ching Ng was born in Hong Kong in 1981. He received the B.Sc. degree in physics from the University of Hong Kong, Hong Kong, in 2004. $\mathrm{He}$ is currently working toward the M.Phil. degree at the University of Hong Kong.

His research interests include organic materials and light-emitting devices (LEDs).

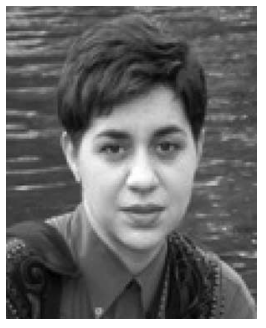

Aleksandra B. Djurišić was born in Vukovar, Croatia, in 1972. She received the Ph.D. degree in electrical engineering from the School of Electrical Engineering, University of Belgrade, Belgrade, Serbia and Montenegro, in 1997.

Since 2003, she has been an Assistant Professor with the Department of Physics, University of Hong Kong, Hong Kong. Her research interests include the optical properties of materials, nanomaterials, wide band gap semiconductors, block copolymers, and optoelectronic devices.

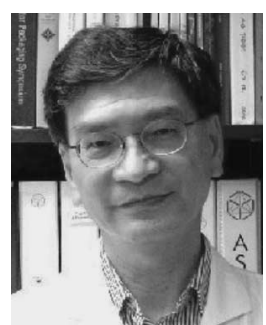

Peter Chow-Kee Liu (S'68-S'90-M'90-SM'01) received the B.Sc. and the M.Sc. degrees from the University of Manitoba, Winnipeg, MB, Canada, and the Ph.D. degree in electrical engineering with specialization in circuit optimization from McMaster University, Hamilton, ON, Canada.

He was a Member of the Scientific Staff with Bell Northern Research (BNR, the former research laboratory of Nortel), Mountain View, CA, where he worked on telecommunication systems and data protocols. He was a Cofounder of a data and satellite communication equipment manufacturing company in the Science-Based Industrial Park, Hsinchu, Taiwan, R.O.C. He joined the Department of Electronic Engineering, Hong Kong Polytechnic University, Hong Kong, as a Principal Lecturer and became an Associate Professor and the Communication Section Head in 1995. Since 2002, he has been an Adjunct Professor with the Chinese University of Hong Kong, Hong Kong. He is also a Guest Professor with the Central South University, Changsha, China. He is currently the Chief Technology Officer of ASM Pacific Technology, Hong Kong, a manufacturer of semiconductor packaging equipment located in Hong Kong and Singapore.

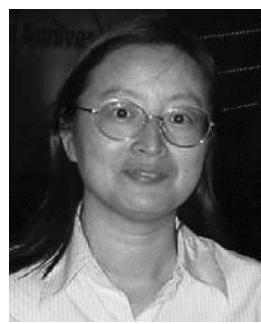

Ming $\mathbf{L i}$ received the $\mathrm{Ph} . \mathrm{D}$. degree in materials science from the University of London, London, U.K., in 1993.

She worked in various universities and institutes in the U.K., Singapore, and Hong Kong before joining ASM Assembly Automation Ltd., Hong Kong, as a Technical Manager. She is the author and coauthor of over 50 peer-reviewed technical papers and international conference papers. Her research interests include packaging technologies for high-power LEDs, flip-chip packaging, package reliability and failure analysis, lead-free solders, and thin-film metallizations.

$\mathrm{Dr}$. $\mathrm{Li}$ is the Chairman for the IEEE CPMT Hong Kong Chapter. 